\title{
Predicting road traffic accidents using artificial neural network models
}

Borja García de Soto PhD, PE

Assistant Professor of Civil Engineering, Division of Engineering, New York University Abu Dhabi, Saadiyat Island, Abu Dhabi, UAE; Tandon School of Engineering, New York University, Brooklyn, NY, USA (corresponding author: garcia.de.soto@nyu.edu)

Andreas Bumbacher MSc

Consultant, Helbling Beratung + Bauplanung, Zurich, Switzerland
Markus Deublein PhD

Project Manager, EBP Schweiz AG, Zollikon, Switzerland

Bryan T. Adey PhD

Professor, Institute of Construction and Infrastructure Management, ETH Zurich, Zurich, Switzerland

As of 2015, Switzerland's road network was among the safest when compared to other European countries. Nonetheless, the endeavour to further decrease the number of traffic accidents and road deaths remains part of the federal agenda. A proper assessment of the relevant risks is, therefore, of utmost importance. This paper presents a methodology for establishing an accident risk prediction model, which can be used as a decision-making tool in infrastructure management. The methodology allows for an appropriate handling of the available data, examines how it can be used to develop models using artificial neural networks (ANNs) and establishes a systematic ANN optimisation process to determine the optimal architecture of the ANN model. The methodology is implemented using data for accident counts on the Swiss national roads from 2009 to 2012. It has been found that ANNs can be used as a viable method to predict the frequency of road accidents. As accident occurrences are relatively rare events, the data are characterised by a large portion of zero observations. This poses a challenge for the training of the ANN. The results show that such models provide reliable results as indicated by the symmetric mean absolute percentage error, ranging from $17 \cdot 5$ to $32 \cdot 7 \%$.

\section{Notation}

AADT annual average daily traffic (veh/d)

$h_{m} \quad$ hidden neuron $m$ in hidden layer of artificial neural network (ANN)

$K \quad$ number of weights in ANN

$l \quad$ length of homogeneous segment $(\mathrm{km})$

$n$ number of observations

o output in output layer of ANN

$t_{i} \quad$ target value for observation $i$

$\widehat{t}_{i} \quad$ output for observation $i$

$v \quad$ exposure $\left(10^{6}\right.$ veh-km-year)

$w_{i j} \quad$ weight between input $i$ and hidden neuron $j$

$w_{j} \quad$ weight between hidden neuron $j$ and output

$X_{i} \quad$ importance for input $i$

$x_{p} \quad$ input variable $p$ in input layer of ANN

$y \quad$ number of accidents in observation period

$\Delta t \quad$ observation period (year)

$\lambda \quad$ average accident rate (acc/( $10^{6}$ veh-km-year))

$\lambda_{i} \quad$ observed (updated) accident rate for segment $i$

$\hat{\lambda}_{i} \quad$ predicted accident rate for segment $i$

\section{Introduction}

In comparison to the roads in other European countries, Swiss roads rank among the safest. The European Transport Safety Council collected accident data from 32 countries, comparing the road deaths per million inhabitants in 2014 (ETSC, 2015a). Malta (24), Sweden (28), the UK (28), Norway (29) and Switzerland (30) recorded the lowest road mortalities; Poland (84), Lithuania (90), Bulgaria (90), Romania (91) and Latvia (106) recorded the highest for 2014. With five deaths per billion vehicle-kilometre between 2012 and 2014, Switzerland is well below an estimated average of 7.37. Lower values were achieved only in Sweden, the British Isles, Norway, Denmark and Finland (ETSC, 2015a). The goal of the EU's 28 member states is clear, and they are committed to 'play their part in cutting road deaths and serious injuries by half by 2020 worldwide' (ETSC, 2015b).

From 2007 to 2013, Swiss roads have had a steady decrease of traffic accidents resulting in injuries of any type (i.e. minor injuries, serious injuries and fatalities). Although these numbers had a slight increase in 2014 and 2015, Swiss roads are considered safe. The general trend for the number of accidents involving fatalities is also decreasing, with the lowest value (229) achieved in 2014 (Figure 1). Nevertheless, an average of 48.59 accidents with personal injury occurred every day in Swiss roads during 2015, or a total of 17736 , of which 238 resulted in fatalities, with a total of 253 deaths (BFS, 2015).

Even with relatively low risks, the Swiss Federal Roads Office (Fedro or Astra in German) strives to further increase road safety. From the perspective of road owners and road infrastructure (safety) managers, the understanding of the factors that contribute to a higher frequency of traffic accidents is of paramount importance. In addition to the three Es - engineering, enforcement and education - used to manage road safety (DFT, 2011), the planning, construction and maintenance of safe roads require in general the consideration of different fields influencing the occurrence of road accidents (e.g. human behaviour, developments in automotive industries and weather). 


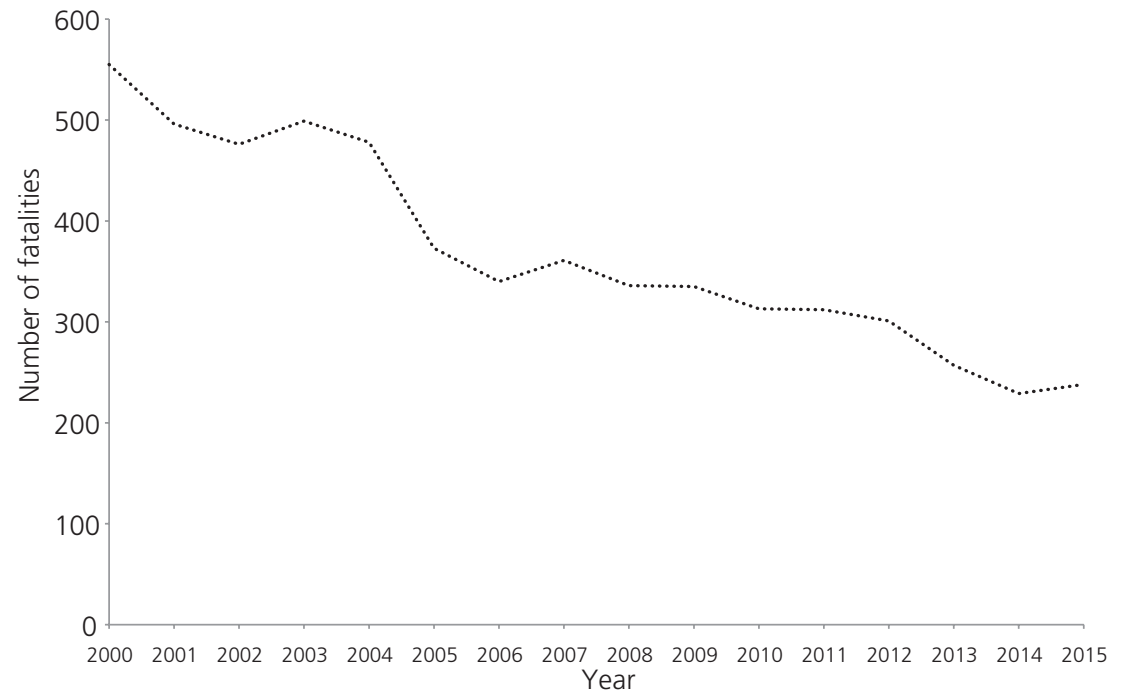

Figure 1. Number of deaths in road accidents in Swiss roads (BFS, 2015)

In this paper, the perspective of road owners and road infrastructure (safety) managers is considered. For them, the most reasonable approach is to estimate infrastructure-related risks and execute risk-reducing interventions on the infrastructure. The use of artificial neural networks (ANNs) is proposed to assist in the estimation of those risks. Models developed using ANNs could be used as a tool to complement the knowledge and experience of road managers in order to support human decision making.

\section{State of the art}

Recent studies for accident risk analysis are based on the Bayesian interpretation of probability (e.g. de Oña et al. (2011, 2013) and El-Basyouny and Sayed (2011)), and several methods have evolved for the development of accident prediction models using Bayesian probabilistic networks (Deublein et al., 2013, 2014, 2015). In addition, the application of ANNs has been investigated. ANNs are well-studied modelling tools that have the ability to map complex non-linear patterns and often non-intuitive relationships between variables. They have proven their effectiveness in various fields of engineering, such as estimation of construction cost ( $\mathrm{Li}, 1995$; Mckim, 1993) or of construction material quantities (García de Soto et al., 2014). This paper is focused on the uses of ANN models.

Some researchers have compared the performance of ANN models to other approaches. Chang (2005) established an ANN model to predict the number of accidents and found it to be a consistent alternative to a negative binomial regression model. Xie et al. (2007) compared three types of modelling techniques, Bayesian ANNs, (backpropagation) ANNs as well as negative binomial regression. They found that both the ANN and the Bayesian ANN model performed better than the negative binomial regression in predicting vehicle accidents. Ali and Bakheit (2011) used ANN models to analyse and predict the road traffic accidents in Sudan. They compared the ANN models with principal component regression models. Their findings showed that although the ANN models had a better fit for the data (as determined by the coefficient of determination), the predictions were very similar with both methods. Sameen and Pradhan (2017) developed a recurrent neural network (RNN) to predict accidents of different severity. The RNN model was compared with multilayer perceptron (MLP) and Bayesian logistic regression (BLR). The RNN model was found to be more accurate than the ANN and BLR models.

A typical single-layer ANN (Figure 2) contains an input layer consisting of $p$ number of input variables plus a bias, a hidden layer consisting of $m$ number of neurons plus a bias and an output layer with the desired output $(o)$. The neurons in the hidden and output layers are often referred to as processing neurons or processing elements. In addition to the normal neurons, there are also bias neurons (e.g. bias 1 and bias 2 in Figure 2), which are used to help ensure that the model has a good fit with existing data. The connections between neurons in the ANNs are used to model the relationships between the neurons - that is, a change in the value of one neuron has an effect on the value of another neuron and, consequently, the value of the output. In addition to these components, an ANN has an activation function, which is used to introduce non-linearity into the model to ensure that there is a better fit between the values predicted by the model and actual data. For a more general insight into the concept of ANNs and their scope of applications, the reader is referred to the works of Hegazy et al. (1994), Bishop (1994), Hill et al. (1994) and Zhang et al. (1998), among others.

Within the domain of civil engineering, ANNs have been used to address function approximation, prediction and optimisation problems (Jain et al., 1996). ANNs are very useful in 


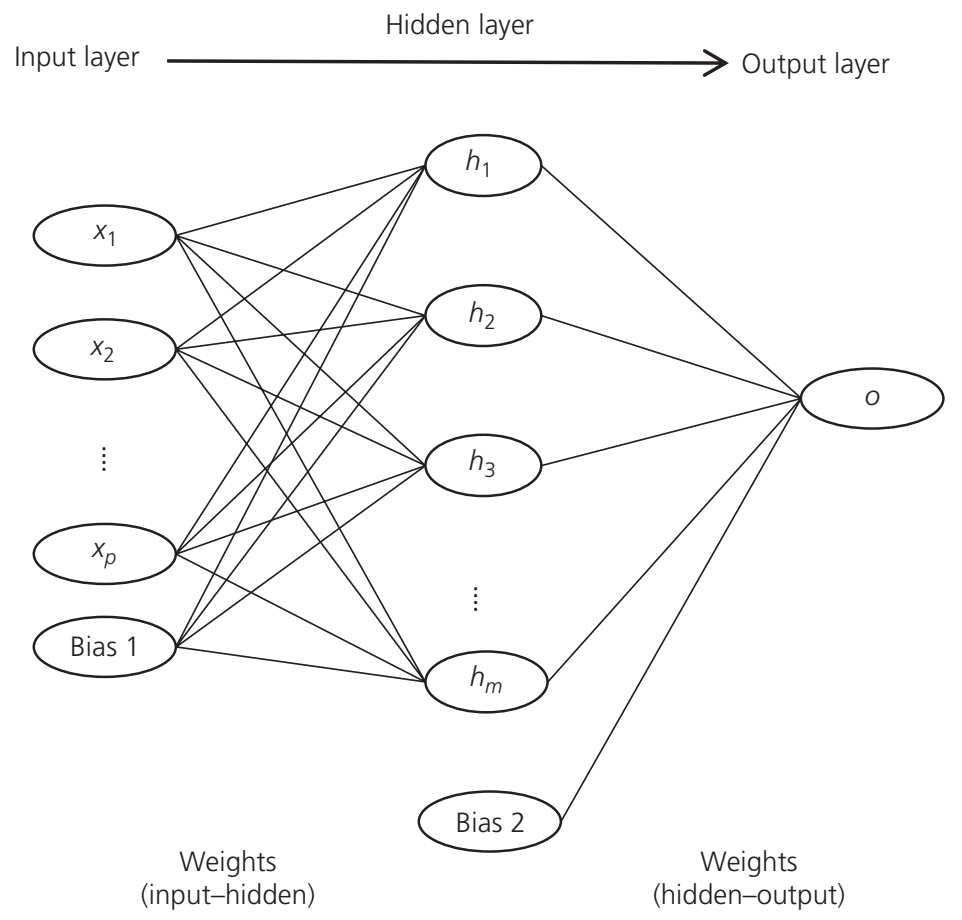

Figure 2. Typical ANN configuration (adapted from García de Soto, 2014).

approximating unknown non-linear functions that depend on a large number of variables, as the use of ANNs eliminates the need to define that function (Kim et al., 2004; Yeh, 1998). Indeed, the use of ANNs was encouraged due to the difficulty of determining the shape of the function in any parametric estimation using regression analysis, in particular complex non-linear ones. Kirby et al. (1997) and Karlaftis and Vlahogianni (2011) discuss the shortcomings of past ANN applications in the field of transportation, such as the choice of inefficient or cumbersome ANN structures, and proposed ways to optimise them.

In past research, various methodologies have been proposed to develop accident risk models. ANNs are among the techniques that have been repeatedly and successfully applied in this area. Delen et al. (2006) used 30358 police-reported accident records that contained motor vehicle crashes from 1995 to 2000 to develop eight binary MLP neural network models with different levels of injury severity (from no injury to fatality) as the dependent variable. Their models assisted to identify important explanatory variables for each dependent variable. Jadaan et al. (2014) developed an accident prediction model analysing the relationship between accidents and parameters affecting them using an ANN. The model produced good results for Jordanian traffic conditions. Ogwueleka et al. (2014) used an ANN model for the analysis and prediction of accident rates in Nigeria. The sigmoid and linear functions were used as activation functions with the feed forward-backpropagation algorithm. The authors indicate that the results using the ANN model outperformed other statistical methods. Alkheder et al. (2017) used data from 5973 traffic accident records that occurred in Abu Dhabi from 2008 to 2013 to develop an ANN model to predict the degree of injury (minor, moderate, severe and death) of road traffic accidents. Their model had an overall prediction performance of $74.6 \%$ when using the testing set.

While ANNs can approximate any function for which they are trained, their structures only provide limited insight about the nature of the approximated function and the interpretation of the results is not straightforward. They have therefore often been criticised for being a black-box technique (e.g. Hegazy et al. (1994) and Boussabaine (1996)). To bypass this issue, researches (Chang, 2005; Delen et al., 2006; Principe et al., 2000) proposed the use of sensitivity analyses to describe the cause and effect relationship between the variables of the ANNs. To address these deficiencies, this paper provides a methodology to develop ANNs in a transparent way and uses sensitivity analysis to determine the influence of the different inputs to the number of accidents. This is useful to infrastructure managers because ANNs developed this way will not only help identify areas of high risk in road networks, but help determine how the different inputs affect their occurrence, hence giving infrastructure managers a support tool to help during the decision-making process and modification of influential parameters within their control (e.g. curvature, speed limit and surface adhesion rating).

\section{Methodology}

This section presents the investigated methodology to develop models to predict road traffic accidents using ANNs for a given 
dataset. The methodology to develop the ANN model consists of several components (Figure 3). Since this type of model is based on a vast amount of data, several components of this methodology deal with the handling and processing of data.

The focus of this section is on the development of ANN models and the different elements used to define the architecture of an $\mathrm{ANN}$, in particular, the number of neurons in the hidden layer. During this task, other elements, such as the choice of the learning algorithm, as well as the type of activation function used, should be evaluated during its implementation. Since these elements are highly influenced by the type of data used, they are addressed in the section headed 'Example'.

\section{Define and determine homogeneous segments}

The definition and determination of homogeneous segments is done in accordance with guidelines given by Deublein et al. (2015: p. 147), which states that 'a homogeneous segment is defined as a road segment in which the values of the considered variables remain constant'. The variables used to determine the homogeneous segments are those that provide an indication of the risk of accidents and that are readily available. Examples of such variables are the annual average daily traffic (AADT), the percentage of heavy good vehicles (HGV), the curvature (Bend), and the slope/gradient (SLP). The values of the risk indicating variables for all homogeneous segments are then considered as raw data.

\section{Preprocess data}

The raw data are preprocessed to determine the input and output variables for the ANN model. The following steps were used.

(a) Determine the exposure for each homogeneous segment (exposure being a function of the length of the homogeneous segment, the observation period and the average daily traffic) expressed in million vehicle-kilometres-year (Equation 4).

(b) Determine the accident rate for each homogeneous segment, expressed in the number of accidents per million-vehiclekilometres (Equation 5).

(c) Weigh each of the homogeneous segments according to their exposure - that is, give more importance to segments with high exposure. (d) Define the input and output variables (weighted and unweighted).

All segments are assigned a weight $w$ defined as the ratio between the segment's exposure $v$ and the mean exposure of all segments $\bar{v}$ rounded to the nearest greater integer. This is done to assign a higher importance to segments with a large number of vehicles as their observed number of accidents is more representative of longterm averages than segments with a small number of accidents. The importance of each segment is represented in the preprocessed dataset by replicating the segments in accordance with their weights (Deublein et al., 2013) - for example, a weighting factor of 2 means that a segment would be replicated once, in the preprocessed dataset.

Since weighting of the segments in this way results in additional data processing effort, the additional benefit obtained from using the weighted against the unweighted datasets need to be considered. This is done by generating both sets of input and output values, conducting the initial analyses using both sets separately and then comparing the results.

Since accident occurrences are relatively rare events, the distribution of accident counts is often denoted by a large majority of zero observations, which translates into analytical difficulties. The very small number of accidents causes the dispersion (i.e. variance) of the counts not to be the same as the mean value (i.e. the sample is overdispersed) (Berk and MacDonald, 2008; Cox, 1983; Dean and Lawless, 1989; Gschloessl and Czado, 2008; Hauer, 2001; Karlis and Meligkotsidou, 2005; Lord and Mannering, 2010). To address overdispersion, the negative binomial regression was applied by assuming that the number of accidents can be represented by a negative binomial distribution (Connors et al., 2013; Deublein et al., 2013; Hauer, 2001; Miaou and Lord, 2003; Mitra and Washington, 2007). The problem of overdispersion is also an issue for the training of ANNs. The accident rates were therefore subjected to an updating process. The updating process proposed by Deublein et al. (2013) was adopted. In addition, it was assumed that the variation of accident frequency could be best described by a negative binomial distribution, which is a compound probability distribution. Essentially, the negative

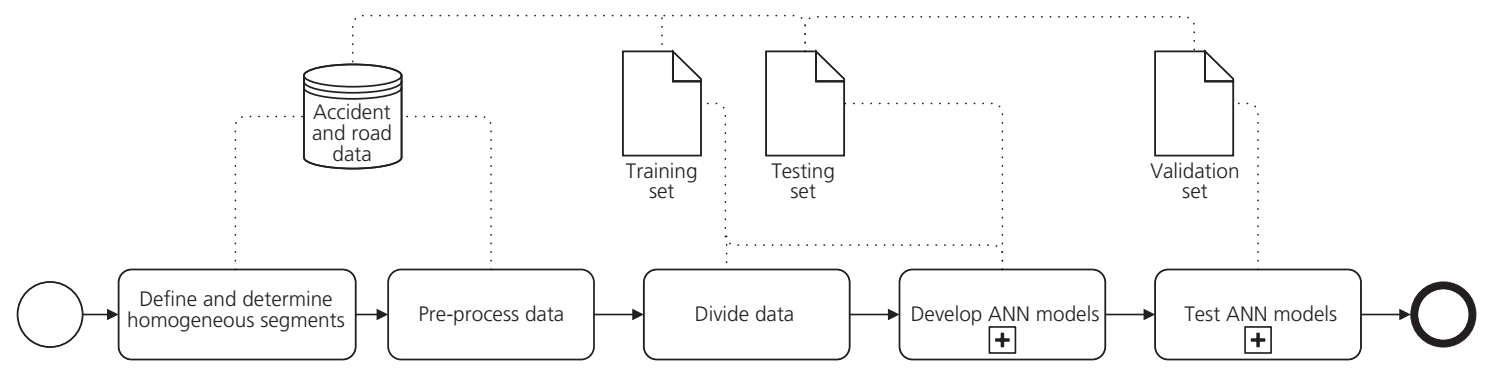

Figure 3. Process for development of ANNs for predicting traffic accidents 
Infrastructure Asset Management

Volume 5 Issue 4
Predicting road traffic accidents using

artificial neural network models

García de Soto, Bumbacher, Deublein and Adey binomial is a Poisson distribution, where the Poisson mean $\lambda$ is in turn a random gamma-distributed variable. For a complete description of the approach, the reader is referred to Deublein et al. $(2013,2014)$ and Gelman et al. (2004).

\section{Divide data}

In order to avoid the overfitting of the model, the data points used to develop the ANN are divided into a training and a testing set. The decision on which ratio should be used needs to be made taking into consideration that $(a)$ the smaller the percentage of data used for training the ANN, the less predictive power it may have and $(b)$ the larger the percentage of data used the less suitable the ANN will be in general situations. A good rule of thumb is an 80:20 division (Hegazy and Ayed, 1998) - that is, $80 \%$ of the data is used for training and the remaining $20 \%$ is used for testing.
In addition, a third set of data, not used for model development, should be put aside for testing the model (refer to the section headed 'Test ANN model').

\section{Develop ANN models}

The developed ANN models are multilayer feed forward ANNs (i.e. no loops in the network) with between three and ten neurons in a single hidden layer and the same number of neurons in the output layer as there are output variables.

The process for the development of the ANN models is shown in Figure 4. It is set up for different road types (e.g. roads (TYP 1) and tunnels (TYP 2)). The first (inner) loop in this process is used to set up and train the ANNs. With each iteration of the loop, the number of neurons in the hidden layer $\left(h_{n}\right)$ are varied from three to ten, as suggested by Blum (1992). The second (outer) loop is

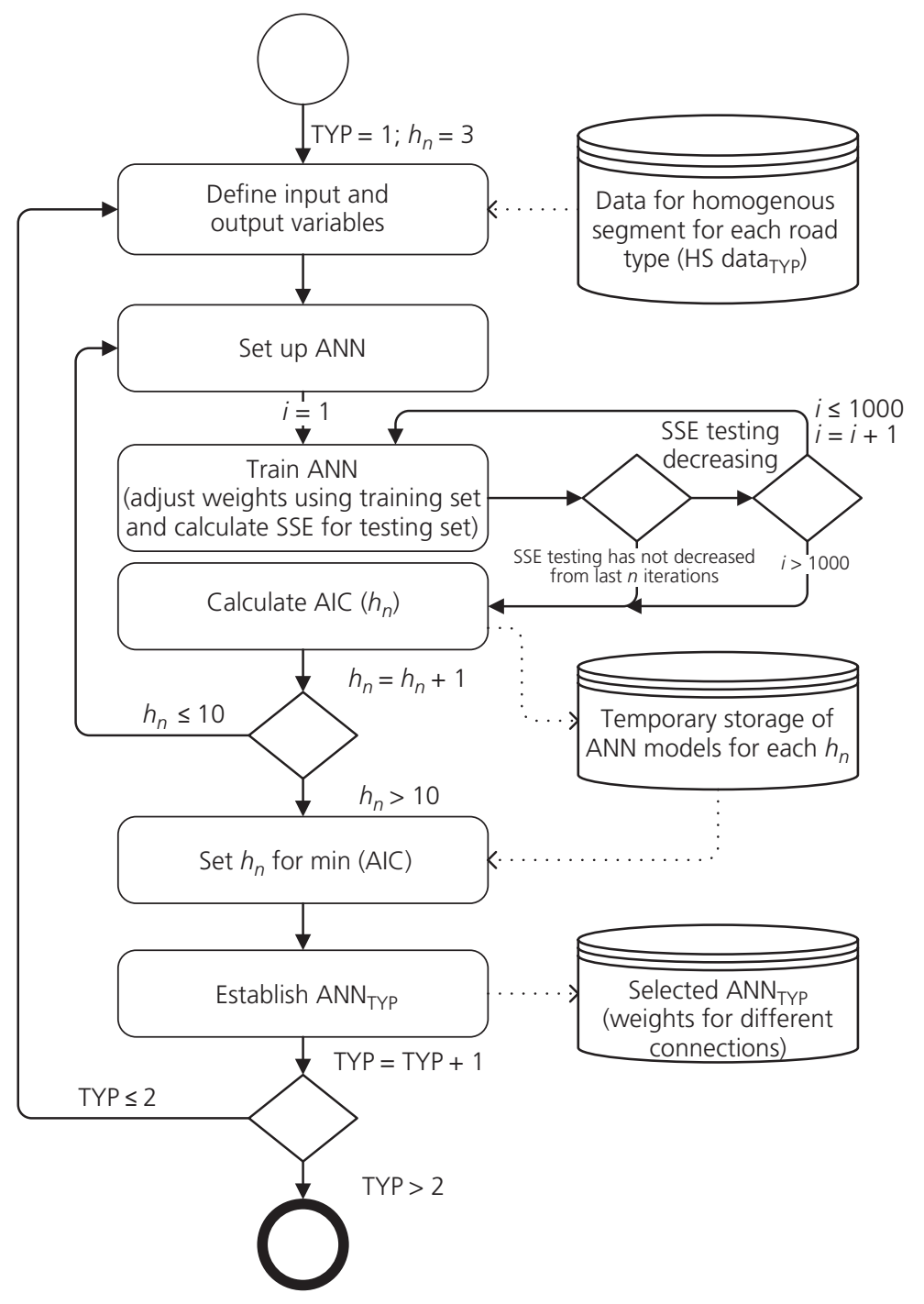

Figure 4. Process for development of ANN for each road type 
used to switch from one object type to the next. This process could be run for each investigated combination of activation function and learning algorithm. Different activation functions and training algorithms were used in the development of the ANN in the section headed 'Example'.

During the training process, the training set is used to determine the weights and biases that are modified using the results of each iteration until the training process ends. To avoid overfitting and ensure the generalisation of the developed models, the testing set is used during the training process as one of two stopping criteria. The performance of the ANN for each iteration is measured using the sum of square errors (SSE) (Equation 1) from the testing set, where the lower the SSE, the better. Therefore, the first stopping criterion evaluates the development of the SSE of the testing set, so that when the SSE does not decrease after a predetermined number $(n)$ of consecutive iterations, the testing process ends. This number $(n)$ should be determined considering the avoidance of getting stuck in local minima. The second stopping criterion ensures that the process does not keep looping indefinitely, so when 1000 iterations are reached, the training process ends.

$$
\text { 1. } \mathrm{SSE}=\sum_{i=1}^{n}\left(\widehat{t}_{i}-t_{i}\right)^{2}
$$

where $n$ is the number of observations; $\widehat{t}_{i}$ is the output for observation $i$; and $t_{i}$ is the target value for observation $i$.

Once the training is completed, the Akaike information criterion (AIC) (Akaike, 1974) is calculated using Equation 2 (Burnham and Anderson, 2002; Burnham et al., 2011). The AIC takes into consideration the number of observations, the number of weights used and the SSE of each ANN. After the different numbers of neurons in the hidden layer have been considered (i.e. a different ANN model has been developed), the AIC is used to select the most accurate model. The ANN with the lowest AIC value is the one with the lowest information loss, hence, predictably, the most accurate model from a set of models (Motulsky and Christopoulos, 2003).

$$
\text { 2. } \mathrm{AIC}=n \ln \left(\frac{\mathrm{SSE}}{n}\right)+2 K
$$

where $n$ is the number of observations and $K$ is the number of weights.

An important characteristic of this metric lies in the fact that, in addition to factoring in square errors, it penalises models with a higher number of estimation parameters (i.e. weights), rewarding simpler models. As asserted by Kirby et al. (1997) and Karlaftis and Vlahogianni (2011), the modelling accuracy should not be the sole determining factor for selecting a methodology. Moreover, the simplicity of the model, as well as its suitability for application, should also be considered.

\section{Test ANN models}

The performance and predictive power of the developed ANN models with the lowest AIC values are tested using the validation dataset. To test the models, the validation dataset is preprocessed with the training and testing sets as indicated in the section headed 'Preprocess data'. Accident rates are determined from the accident frequencies and updated to assign accident rates to homogeneous segments where observations were not collected (Deublein et al., 2014). During this process, the bias towards zero observations was eliminated by determining accident rates from the validation dataset and assuming that the negative binomial distribution was applicable (Deublein et al., 2013, 2014, 2015).

The metric used to evaluate the developed models was the mean absolute percentage error $\left(M_{\mathrm{APE}}\right)$, with the correction proposed by Armstrong (1985) to account for the bias of the $M_{\mathrm{APE}}$ that favours under- or overestimations. Here, the absolute difference between the actual and estimated amount, divided by the average of the actual and the estimated amount, is shown in Equation 3. This correction essentially puts the same penalty on a prediction that is half of the actual value as on one that is double the actual value.

$$
\text { 3. Symmetric } M_{\mathrm{APE}}=\frac{1}{n} \times \sum_{i=1}^{n}\left[\frac{\left|\widehat{\lambda}_{i}-\lambda_{i}\right|}{(1 / 2)\left(\hat{\lambda}_{i}+\lambda_{i}\right)}\right]
$$

where $n$ is the number of observations (segments); $\widehat{\lambda}_{i}$ is the predicted accident rate for segment $i$; and $\lambda_{i}$ is the observed (updated) accident rate for segment $i$.

The output of the ANN model (i.e. predictions) is then directly comparable to the values of the validation set (i.e. observations). Figure 5 shows how the different types of accident data are linked to each other and at which level the validation of the model can be made.

\section{Example}

The methodology to develop ANNs in order to predict traffic accidents was tested using the same dataset as used by Deublein et al. $(2013,2014,2015)$. The dataset, made available by Fedro, contains information on accident occurrences and traffic volume on the Swiss national road network from the years 2009-2012. The accident data points are specific to the location on the road network, used to describe certain traffic and road characteristics. The severity of the accidents (i.e. accident type) was broken down into three categories rating the degree of the resulting personal damage - that is, light injuries (LIN), severe injuries (SIN) and fatalities (FAT). The dataset from 2010 to 2012 was more complete than the one from 2009. Therefore, the dataset from 2010 to 2012 was used for model development, and the dataset 


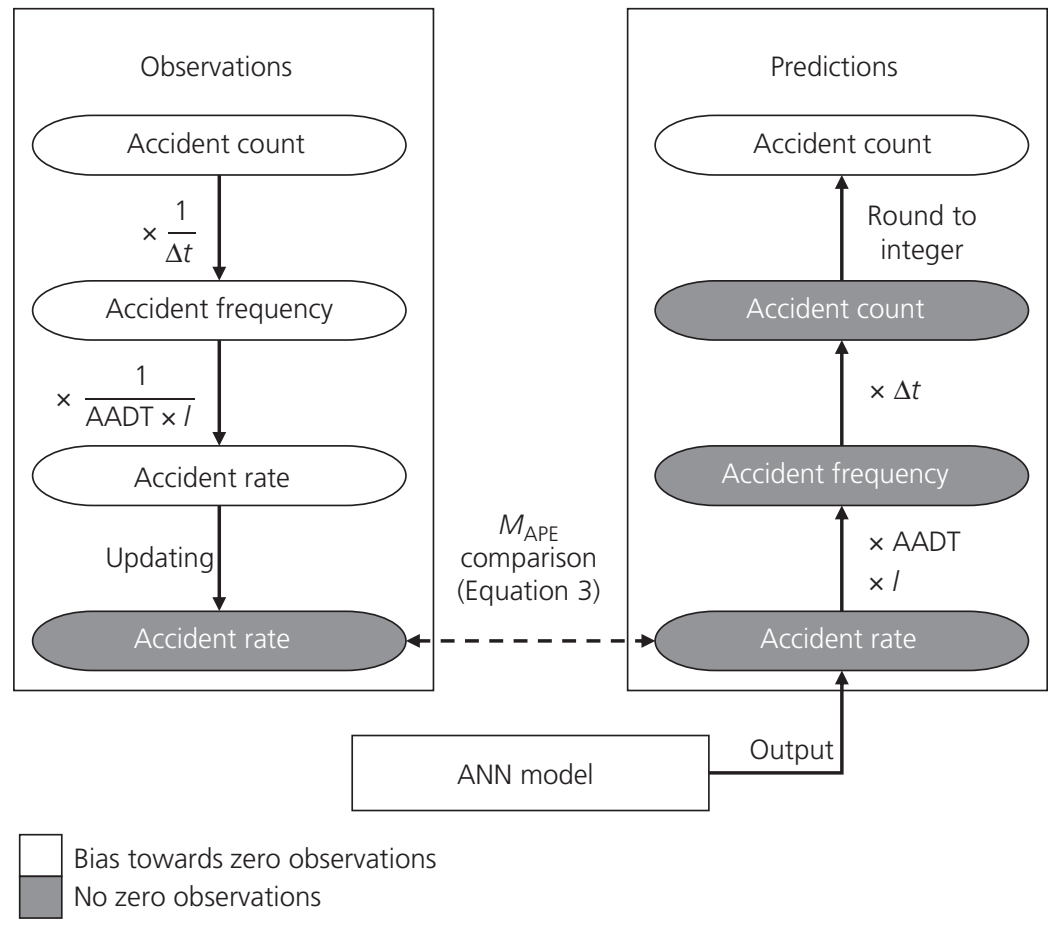

Figure 5. Types of accident data processed in both the level of observations and the level of predictions

for 2009 was used to test the model. It was assumed that the input variables had remained constant from 2009 to 2012 regarding the trend of injuries recorded.

MatLab was used to develop the ANN models. MatLab offers a wide range of in-built functions and tools (e.g. the 'neural network toolbox' provides functions and apps for designing, implementing, visualising and simulating ANNs), making the development of ANNs efficient and yet allowing for customisation and control.

\section{Define and determine homogenous segments}

The road network (total length of $3642 \mathrm{~km}$ for both driving directions) encompassed by the database was divided into sections with the same road and traffic characteristics (i.e. homogeneous segments). The data used for modelling were from the years 2010 to 2012 and include 13109 homogeneous segments.

\section{Preprocess data}

The homogenous segments were further evaluated to determine their exposure (measured as million vehicle-kilometres-year) and accident rate (measured as the number of accidents per million vehicle-kilometres-year). The homogeneous segments were weighted according to their exposure.

Essentially, all segments are assigned a weight $w$ defined as the ratio between the segment's exposure $v$ and the mean exposure of all segments $V$ rounded to the nearest greater integer. The segments were then replicated in the dataset such that the number of copies was equal to the weight, increasing the number of data points from 13109 to 21325 .

\section{Inputs}

The choice of input variables was based on expert opinion and previous research (Deublein et al., 2014, 2015). In particular, they quantify the volume of traffic and a range of road characteristics. The information from the homogeneous segments used as the input of the ANN model is shown in Table 1.

$I_{2}$ and $I_{4}$ are grades evaluating the condition of the road surface. $I_{2}$ rates the longitudinal evenness, whereas $I_{4}$ rates the adhesion (roughness) of the surface. The integers range from 0 to 2 (good), 2 to 4 (acceptable) and 5 (bad), making $I_{2}$ and $I_{4}$ discrete variables. The division of the road network into homogeneous segments, however, does not equate to the sections for which one specific grade applies. Therefore, the grades were adjusted to allow for values between the integers. The road type indicator was used to differentiate between open road and tunnels.

\section{Outputs}

The purpose of the model was the prediction of accidents; therefore, the output variables used for training were indicators of accident occurrence. The dataset divided into homogeneous segments included the number of accidents according to their consequences of personal damage observed in each segment (LIN, SIN and FAT). 
Table 1. Input variables used for model development

\begin{tabular}{llclrr} 
Variable & \multicolumn{1}{c}{ Description } & Unit & Type & Low level & High level \\
AADT & Annual average daily traffic & veh/d & Continuous & 10000 & 50000 \\
HGV & Percentage of heavy traffic & $\%$ & Continuous & 5 & 25 \\
RAD & Average curve radius & $m$ & Continuous & 1000 & 8000 \\
SLP+ & Mean positive slope & $\%$ & Continuous & 0 & 3 \\
SLP- & Mean negative slope & $\%$ & Continuous & 0 & 3 \\
SPD & Signalised speed limit & $\mathrm{km} / \mathrm{h}$ & Categorical & 80 & 2 \\
LAN & Number of lanes per direction & - & Categorical & 2 & 3 \\
$I_{2}$ & Longitudinal evenness rating & - & Continuous & 2 & 4 \\
$I_{4}$ & Surface adhesion rating & - & Continuous & Open road & Tunnel
\end{tabular}

Since the homogeneous segments were defined based on their characteristics, the lengths of the sections have a significant variation. For that reason, the chances of an accident occurring in a particular segment were biased towards its length (i.e. the longer the segment, the higher the probability of an accident occurring on that segment); therefore, the frequency of accidents could not be used as an output variable. This problem was circumvented by determining an accident rate $(\lambda)$ (Equation 4$)$. $\lambda$ values can then be normalised with respect to the segment's exposure to traffic $(v)$ (Equation 5) (Deublein et al., 2014).

4. $\lambda=\frac{y}{v}$

5. $v=l \Delta t \mathrm{AADT}$

where $\lambda$ is the average accident rate (acc/( $10^{6}$ veh-km-year) $) ; y$ is the number of accidents in observation period; $v$ is the exposure ( $10^{6}$ veh-km-year); $l$ is the length of homogeneous segment $(\mathrm{km})$; $\Delta t$ is the observation period (year); AADT is the annual average daily traffic (veh/d).

For the observation period considered, $80 \%$ of the homogeneous segments had no occurrences of resulting in personal damage accidents. The percentages of zero observations specific to the accident type amounted to $82 \cdot 6 \%$ for LIN, $96 \cdot 2 \%$ for SIN and $99 \cdot 5 \%$ for FAT. These rates do not refer to the total length of the road network but, instead, to the number of homogeneous segments as the model treats each segment equally, regardless of its length. These low occurrences were expected as indicated by other researchers (e.g. Hauer (2001), Mitra and Washington (2007), Connors et al. (2013) and Deublein et al. (2013)) and treated accordingly.

After the updating process (i.e., to account for over-dispersion), the accident rates for the three accident types (i.e. severity levels) were non-zero values attributed to each homogeneous segment.

\section{Input modes}

The performance of the ANN model depends not only on the data used for its development, but also in how those data are fed into the model. As such, the determination of the form in which the data are entered was further analysed.

Different modes where used to test the predictive power of the ANN being developed. The characteristics of the two modes (modes 1 and 2) are summarised in Table 2. In essence, mode 2 includes some sort of modification of the preprocessed data, which was used in its raw (i.e. original) form. The evaluation of each of the different modes was used in the process shown in Figure 4. This constituted one of the main characteristics of the determination of the model.

\section{Develop ANN models}

Each mode was used in the process for developing the ANN model (Figure 4). The input data were entered according to the combinations shown in Table 2 - that is, discrete or continuous input variables, accident rates being unchanged or taken into

Table 2. Mode characteristics to define input and output variables

\begin{tabular}{|c|c|c|}
\hline & Mode 1 & Mode 2 \\
\hline Discrete variables & Can be used as input variables of the ANN (general) & $\begin{array}{l}\text { Can be used to train two or more separate ANNs depending } \\
\text { on the chosen variable type (e.g. road type: (a) open road or } \\
\text { (b) -tunnel) (specific) }\end{array}$ \\
\hline $\begin{array}{l}\text { Homogeneous } \\
\text { segments }\end{array}$ & $\begin{array}{l}\text { Can have a balanced influence on the training of the } \\
\text { ANN (balanced) }\end{array}$ & $\begin{array}{l}\text { Can be weighted and replicated according to their exposure } \\
\text { (weighted) }\end{array}$ \\
\hline Input variables & $\begin{array}{l}\text { Can be introduced in their original (e.g. continuous) } \\
\text { form (continuous) }\end{array}$ & Can be discretised to fixed steps (discrete) \\
\hline Output values & $\begin{array}{l}\text { Can be introduced in their original (e.g. continuous) } \\
\text { form (output) }\end{array}$ & Can be introduced as logarithmised output values (in(output)) \\
\hline
\end{tabular}


Infrastructure Asset Management

Volume 5 Issue 4
Predicting road traffic accidents using

artificial neural network models

García de Soto, Bumbacher, Deublein and Adey
Table 3. Variables for development of ANN models

\begin{tabular}{lll}
\hline ANN element & \multicolumn{2}{c}{ Variable } \\
\hline Activation function & Hyperbolic tangent & Logistic function \\
Learning algorithm & Resilient backpropagation & Delta rule
\end{tabular}

logarithm and weighted or non-weighted number of road segments. For each mode type, different activation functions and learning algorithms were evaluated. In addition, the road type (TYP) was further evaluated by considering it as an input variable or by developing dedicated models for each road type.

The activation functions selected to introduce non-linearity to the ANN were the hyperbolic tangent and the logistic function (i.e. the inverse of the natural logit function). These activation functions were chosen because they have proven to yield good results (Hegazy et al., 1994). The learning algorithms evaluated were the resilient backpropagation algorithm and the delta rule as suggested by Beale et al., 2014. These are the most common learning algorithms for supervised ANNs. For each mode, the different elements evaluated for the development of the ANN models are summarised in Table 3 .

From this evaluation, two distinct ANNs for either road type were developed. They have the same basic configuration, with the exception of the number of neurons in the hidden layer $\left(h_{n}\right)$, which was determined from the evaluation of the AIC. The main characteristic of the ANNs are

- one ANN for each road type (open road and tunnel)

- homogeneous segments weighted and replicated according to their exposure

- continuous (non-discretised) input variables

- logarithmised output values (accident rates).

Figure 6 shows the overall configuration of the ANN models. The input variables of all segments were separated according to the road type and either fed to ANN 1 or ANN 2. $h_{n}$ indicates the number of neurons in the hidden layer for each ANN. The output of the ANNs - that is, the accident rates $\lambda_{\mathrm{k}}$ - were then used to determine the predicted accident frequencies.

The weights between the input and hidden layer and the hidden layer and output for the ANN for open roads are shown in Tables 4 and 5, respectively. One of the main challenges of ANNs is to understand how the input affects the output. This is not obvious by just looking at the weights, as opposed to - for instance, the coefficients from regression models. However, the weights can be further used to determine a ranking (i.e. importance) of the variables to get a better idea of which ones have the most impact to the output.

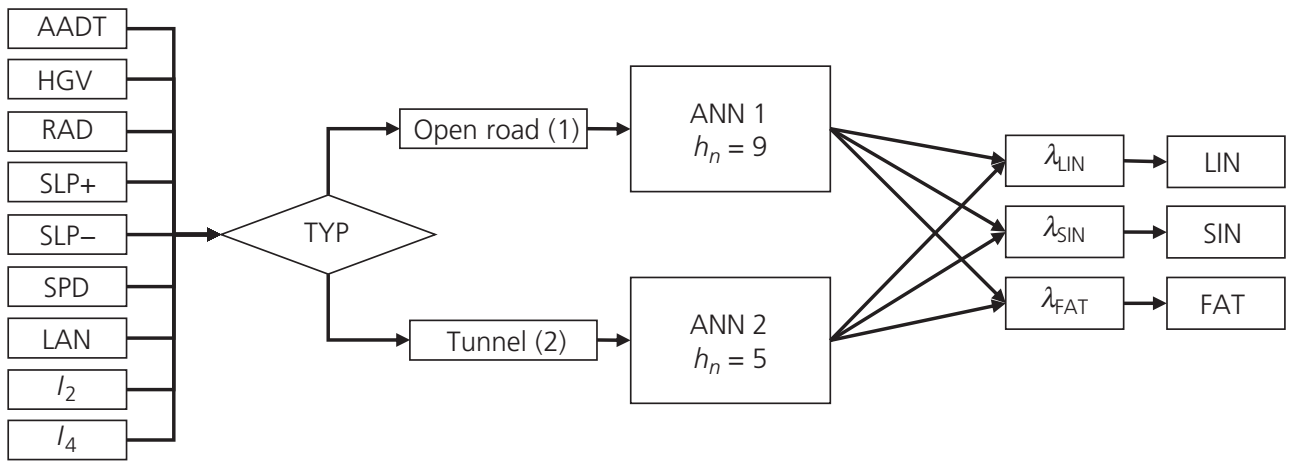

Figure 6. Schematic chart of the information flow through the model

Table 4. Weights between input and hidden layers for ANN for open roads (TYP $=1$ )

\begin{tabular}{lrrrrrrrrrr} 
Hidden neuron & AADT & HGV & RAD & \multicolumn{1}{c}{ SLP+ } & \multicolumn{1}{c}{ SLP- } & \multicolumn{1}{c}{ SPD } & \multicolumn{1}{c}{ LAN } & \multicolumn{1}{c}{ Bias } \\
$n_{1}$ & -0.151 & -0.321 & -0.775 & 0.773 & -0.553 & 0.072 & 0.458 & -0.002 & -0.465 & 1.539 \\
$n_{2}$ & -0.066 & -0.234 & -0.811 & -0.613 & 0.459 & 0.135 & 0.338 & 0.152 & 0.303 & 1.488 \\
$n_{3}$ & 0.011 & 0.176 & 0.951 & 0.188 & 0.069 & -0.106 & -0.384 & -0.227 & -0.284 & -1.286 \\
$n_{4}$ & -0.013 & 0.535 & 1.083 & 0.963 & 0.358 & 0.058 & -0.458 & -0.329 & -0.029 & -1.046 \\
$n_{5}$ & -0.314 & -0.179 & 0.978 & 1.067 & 0.415 & -0.361 & -0.331 & 0.552 & -0.940 & -0.179 \\
$n_{6}$ & 0.014 & 1.724 & -2.247 & -0.494 & -0.078 & 1.511 & 3.584 & -2.303 & -0.902 & 5.149 \\
$n_{7}$ & 0.754 & 0.692 & 0.720 & 0.384 & 0.331 & -0.067 & -0.523 & 0.240 & 0.776 & -0.057 \\
$n_{8}$ & -0.046 & -0.295 & -1.054 & -0.421 & -0.036 & 0.038 & 0.394 & 0.384 & 0.013 & 1.345 \\
$n_{9}$ & -0.382 & 2.474 & -3.972 & -0.563 & -1.002 & 2.669 & 5.636 & -3.562 & 0.184 & 8.402
\end{tabular}


Table 5. Weights between hidden layer and output for ANN for open roads (TYP $=1$ )

\begin{tabular}{lcccccccccc} 
Output & $\boldsymbol{n}_{\mathbf{1}}$ & \multicolumn{1}{c}{$\boldsymbol{n}_{\mathbf{2}}$} & $\boldsymbol{n}_{\mathbf{3}}$ & \multicolumn{1}{c}{$\boldsymbol{n}_{\mathbf{4}}$} & $\boldsymbol{n}_{\mathbf{5}}$ & $\boldsymbol{n}_{\mathbf{6}}$ & $\boldsymbol{n}_{\mathbf{7}}$ & $\boldsymbol{n}_{\mathbf{8}}$ & $\boldsymbol{n}_{\mathbf{9}}$ & Bias \\
LIN & -0.459 & -0.159 & -0.679 & -0.730 & 0.092 & 0.939 & 0.073 & -0.860 & 0.192 & -0.843 \\
SIN & 0.958 & 0.706 & 0.202 & 0.156 & 0.158 & 0.354 & 0.759 & 0.107 & 0.167 & -0.941 \\
FAT & 0.023 & -0.084 & 0.016 & 0.634 & 0.095 & 0.119 & 0.306 & 0.991 & 0.362 & -0.419
\end{tabular}

Table 6. Weights between input and hidden layer for ANN for tunnels (TYP = 2)

\begin{tabular}{lrlllllrrrr} 
Hidden neuron & AADT & HGV & RAD & SLP+ & SLP- & \multicolumn{1}{c}{ SPD } & \multicolumn{1}{c}{ LAN } & \multicolumn{1}{c}{$\boldsymbol{I}_{\mathbf{2}}$} & \multicolumn{1}{c}{$\boldsymbol{I}_{\mathbf{4}}$} & \multicolumn{1}{c}{ Bias } \\
$n_{1}$ & -0.222 & 0.054 & -0.833 & -0.366 & 0.227 & -0.177 & 0.519 & 0.567 & -0.352 & 1.527 \\
$n_{2}$ & 0.901 & -0.056 & -1.242 & -0.652 & -1.390 & 4.196 & -0.903 & -0.944 & -0.129 & 0.728 \\
$n_{3}$ & -0.579 & -0.204 & -1.242 & -0.868 & -0.089 & -0.256 & 0.564 & 0.707 & -0.418 & 0.555 \\
$n_{4}$ & 0.053 & -0.409 & -1.091 & -0.513 & -0.058 & -0.214 & 0.528 & 0.523 & 0.105 & 0.953 \\
$n_{5}$ & 1.371 & 1.509 & 1.282 & 1.623 & -0.387 & -0.731 & -2.605 & 3.427 & -1.680 & -3.180
\end{tabular}

The weights between the input and hidden layer and the hidden layer and output for the ANN for tunnels are shown in Tables 6 and 7 , respectively.

The importance of each input can be estimated using the sum product of the raw input-hidden and hidden-output connection weights as shown in Equation 6. This is known as the connection weight approach (Olden and Jackson, 2002).

6.

$$
X_{i}=\sum_{j=1}^{m}\left(w_{i j} \times w_{j}\right)
$$

where $X_{i}$ is the importance for input $i ; w_{i j}$ is the weight between input $i$ and hidden neuron $j$; and $w_{j}$ is the weight between hidden neuron $j$ and output.

For the open road models, the number of lanes, the average curve radius and the percentage of heavy traffic had the highest importance for all accident types. For the tunnel models, the longitudinal evenness rating, number of lanes and surface adhesion rating had the highest importance for all accident types.

\section{Test ANN models}

Accident data from 2009 were used to test the ANN model in terms of predictive performance, as well as its generalisation properties. These data were not used in any way for the development of the model.

The validation data contained 8984 homogeneous segments and were used in its raw form - that is, sectioned into the segments

Table 7. Weights between hidden layer and output for ANN for tunnels $($ TYP $=2)$

\begin{tabular}{lrrrccc} 
Output & \multicolumn{1}{c}{$\boldsymbol{n}_{\mathbf{1}}$} & \multicolumn{1}{c}{$\boldsymbol{n}_{\mathbf{2}}$} & \multicolumn{1}{c}{$\boldsymbol{n}_{\mathbf{3}}$} & $\boldsymbol{n}_{\mathbf{4}}$ & \multicolumn{1}{c}{$\boldsymbol{n}_{\mathbf{5}}$} & \multicolumn{1}{c}{ Bias } \\
LIN & -0.019 & -0.053 & -0.245 & 0.354 & -0.328 & -0.132 \\
SIN & -0.621 & -0.063 & 0.082 & 0.339 & -0.621 & -0.010 \\
FAT & 0.015 & -0.021 & -0.080 & 0.173 & -0.424 & -0.406
\end{tabular}

with the corresponding input variables and the accident counts for all levels of severity (i.e. accident type). The input variables were fed into the models with the same units for which the model was trained.

Table 8 shows the results of symmetric $M_{\mathrm{APE}}$ (Equation 3) on the validation dataset.

\section{Model comparison}

The performance of the ANN model was compared to the Bayesian probabilistic model (i.e. Bayesian network (BN)) developed by Deublein et al. $(2014,2015)$. The comparison was based on the errors obtained from the predicted accident rates using the data from 2010 to 2012 .

Table 9 shows the systematic $M_{\mathrm{APE}}$ from each model for different accident types. For light (LIN) and severe injuries (SIN), both models have similar performance. However, for fatalities (FAT), the ANN model outperformed the BN (30.0 against $51 \cdot 8 \%$ for $\mathrm{ANN}$ and $\mathrm{BN}$, respectively).

It was also found that the $\mathrm{BN}$ model had a higher tendency to overestimate accident frequencies when compared to the ANN model (Table 10).

The modelling accuracy should not be the only determining factor for selecting a methodology. The selection should also take into consideration the suitability of the model and methodology for application. In this study - for example, the BN model did not perform as well with regard to the errors as the neural network model, but the BN model does provide a stochastic representation

Table 8. $M_{\text {APE }}$ (symmetric) for predicted against the observed (updated) accident rates for all levels of severity using 2009 validation data

\begin{tabular}{llll|}
\hline Time period & \multicolumn{3}{c}{$\mathbf{2 0 0 9}$} \\
\hline Accident type & LIN & SIN & FAT \\
Symmetric $M_{\text {APE: }} \%$ & 17.5 & 32.7 & 30.6
\end{tabular}


Table 9. $M_{\text {APE }}$ (symmetric) for the predicted against the observed (updated) accident rates for both the ANN model and the BN model using the 2010-2012 data

\begin{tabular}{|c|c|c|c|c|c|c|}
\hline Model & & ANN & & & BN & \\
\hline Accident type & LIN & SIN & FAT & LIN & SIN & FAT \\
\hline Symmetric $M_{\mathrm{APE}}: \%$ & $22 \cdot 40$ & $27 \cdot 00$ & 30.00 & $21 \cdot 80$ & $27 \cdot 50$ & $51 \cdot 80$ \\
\hline
\end{tabular}

Table 10. Percentages of overestimated accident frequencies for all levels of severity

\begin{tabular}{lllllll} 
& \multicolumn{3}{c}{ ANN } & \multicolumn{3}{c}{ BN } \\
\hline Accident type & LIN & SIN & FAT & LIN & SIN & FAT \\
Ratio of overestimation: \% & 36.7 & 28.8 & 19.4 & 80.9 & $82 \cdot 1$ & 98.5
\end{tabular}

of the variable combinations, which may be more desirable. An overview of the different features of accident prediction models using $\mathrm{ANN}$ and $\mathrm{BN}$ is given in Table 11. The information provided in this table is not meant to be exhaustive, but based on the specific models developed by the authors in this study.

\section{Conclusion and outlook}

This paper presents a methodology for establishing an accident risk prediction model using ANNs. The developed models can be used as a decision-making tool in infrastructure management to identify locations of a road network where the number of accidents is expected to be high and to be able to concentrate on the factors causing that.

The performance of the ANN model was not significantly affected by the type of activation function or learning algorithm used, but by the input mode of the data and the size of the hidden layer (i.e. the number of neurons in the hidden layer). It was also found that road type-specific ANN models provided better results than ANN models that used the road type as an input neuron. Marginal improvements were observed with the resilient backpropagation, when compared to the delta rule. The resilient backpropagation algorithm also performed better in terms of computational speed. Similarly, the hyperbolic tangent was favoured for all ANNs and proved to work more efficiently in combination with the resilient backpropagation when compared to the logistic function.

The performance of the ANN model varied depending on the accident type. The results using the validation data (from 2009) show that the predictions for accidents with severe injuries and fatalities (symmetric $M_{\mathrm{APE}}$ of 32.7 and $30.6 \%$, respectively) were not as good as those for accidents with light injuries (symmetric $M_{\mathrm{APE}}$ of $17 \cdot 5 \%$ ). When comparing the predictions using the data from 2010 to 2012, it was found that the ANN and BN models had similar performance for light injury accidents (symmetric $M_{\mathrm{APE}}$ of 22.4 and $21.8 \%$ for $\mathrm{ANN}$ and $\mathrm{BN}$, respectively) and for severe injury accidents (symmetric $M_{\mathrm{APE}}$ of $27 \cdot 0$ and $27 \cdot 5 \%$ for ANN and BN, respectively). However, the estimation of accidents with fatalities was more accurate when using the ANN models (symmetric $M_{\mathrm{APE}}$ of 30.0 and $51.8 \%$ for $\mathrm{ANN}$ and $\mathrm{BN}$, respectively). This discrepancy is most likely due to the limited number of observations of accidents with severe injuries and fatalities, which have a direct effect on the mapping between the inputs and the outputs during the training of the ANNs.

Table 11. Qualitative comparison for different features of ANNs and BNs

\begin{tabular}{|c|c|c|}
\hline Feature & ANN & BN \\
\hline Assumptions & $\begin{array}{l}\text { No need for predefined underlying relationship between } \\
\text { the variables }\end{array}$ & $\begin{array}{l}\text { No need for predefined underlying relationship between the } \\
\text { variables. Prior (e.g. experts) definitions of casual variable } \\
\text { relationships can be done for structuring the network }\end{array}$ \\
\hline Encoding & $\begin{array}{l}\text { Deterministic mapping between a set of input variables and } \\
\text { output variables }\end{array}$ & $\begin{array}{l}\text { Deterministic or probabilistic distributions for all combinations } \\
\text { of variables }\end{array}$ \\
\hline $\begin{array}{l}\text { Network } \\
\text { structure }\end{array}$ & Neurons in consecutive layers linked by connection weights & Conditional probability tables \\
\hline Neurons/nodes & Number of hidden neurons determined through heuristics & $\begin{array}{l}\text { Number of nodes dependent on number of input, intermediate } \\
\text { and output variables }\end{array}$ \\
\hline $\begin{array}{l}\text { Variable } \\
\text { dependence }\end{array}$ & $\begin{array}{l}\text { The dependence between variables is not apparent from } \\
\text { the structure }\end{array}$ & $\begin{array}{l}\text { Structure provides complete information on the conditional } \\
\text { interdependence of the variables }\end{array}$ \\
\hline $\begin{array}{l}\text { Categorical } \\
\text { variables }^{a}\end{array}$ & $\begin{array}{l}\text { Are assigned numerical values, adding an arbitrary } \\
\text { topology }\end{array}$ & No influence on topology \\
\hline Before learning & Prior network uses random weights & $\begin{array}{l}\text { Prior network can be completely non-informative or } \\
\text { informative (e.g. when coefficients of regression analysis or } \\
\text { information from literature/experts are used) }\end{array}$ \\
\hline
\end{tabular}

${ }^{a}$ In the proposed ANN model, this was avoided by developing an ANN model for each road type (open roads and tunnels) 
Although it was found that the ANN model resulted in lower symmetric $M_{\mathrm{APE}}$ than the $\mathrm{BN}$ model, this alone should not be used to state that one model is better than another. For example, unlike the ANN models, the $\mathrm{BN}$ models give probabilistic estimates of the number of accidents, which may be something more useful in decision-making in infrastructure management.

The amount and quality of data available for model development is always a challenging issue, but having this kind of models in place sets the precedence for road authorities to organise and collect their data in a more structured and systematic way. This will lead to a dataset with more years of observations, which will ultimately help improve the predicting accuracy of the model.

Future work in this area should be focused on trying to reduce the errors in prediction even further - for example, by developing other options to overcome overdispersion and extending the work to cover other non-infrastructure factors - for example, driver age, experience, condition and vehicle factors. Any future work should also give serious consideration to the coming automation of vehicles, which will drastically change how and why accidents occur.

\section{Acknowledgements}

Special thanks are given to Dr Anja Simma and Mr Gerhard Schuwerk and their team at the Swiss Federal Roads Office for providing the data used in this study.

\section{REFERENCES}

Akaike H (1974) A new look at the statistical model identification. In Selected Papers of Hirotugu Akaike. Springer Series in Statistics (Perspectives in Statistics) (Parzen E, Tanabe K and Kitagawa G (eds)). Springer, New York, NY, USA, pp. 215-222.

Ali GA and Bakheit CS (2011) Comparative analysis and prediction of traffic accidents in Sudan using artificial neural networks and statistical methods. Proceedings of the 30th South African Transport Conference (SATC 2011). Document Transformation Technologies, Centurion, South Africa, pp. 202-214

Alkheder S, Taamneh M and Taamneh S (2017) Severity prediction of traffic accident using an artificial neural network. Journal of Forecasting 36(1): 100-108, https://doi.org/10.1002/for.2425.

Armstrong JS (1985) Long-Range Forecasting: from Crystal Ball to Computer. Wiley-Interscience, New York, NY, USA.

Beale M, Hagan M and Demuth H (2014) Neural Network Toolbox TM User's Guide. MATLAB R 2014a. The MathWorks, Inc., Natick, MA, USA.

Berk R and MacDonald JM (2008) Overdispersion and Poisson regression. Journal of Quantitative Criminology 24(3): 269-284, https://doi.org/ 10.1007/s10940-008-9048-4.

BFS (Bundesamt für Statistik) (2015) Strassenverkehrsunfälle mit Personenschaden und Verunfallte nach Kanton. BFS, Neuchâtel, Switzerland. See http://www.bfs.admin.ch/bfs/portal/de/index/themen/ 11/01/new/nip_detail.html?gnpID=2015-218 (accessed 29/05/2015) (in German).

Bishop CM (1994) Neural networks and their applications. Review of Scientific Instruments 65(6): 1803-1832, https://doi.org/10.1063/1. 1144830 .

Blum A (1992) Neural Networks in C++: an Object-Oriented Framework for Building Connectionist Systems. Wiley, Hoboken, NJ, USA.
Boussabaine $\mathrm{AH}$ (1996) The use of artificial neural networks in construction management: a review. Construction Management \& Economics 14(5): 427-436, https://doi.org/10.1080/014461996373296.

Burnham KP and Anderson DR (2002) Model Selection and Multimodel Inference: A Practical Information-Theoretic Approach. Springer, London, UK.

Burnham KP, Anderson DR and Huyvaert KP (2011) AIC model selection and multimodel inference in behavioral ecology: some background, observations, and comparisons. Behavioral Ecology and Sociobiology 65(1): 23-35, https://doi.org/10.1007/s00265-010-1029-6.

Chang LY (2005) Analysis of freeway accident frequencies: negative binomial regression against artificial neural network. Safety Science 43(8): 541-557, http://dx.doi.org/10.1016/j.ssci.2005.04.004.

Connors RD, Maher M, Wood A, Mountain L and Ropkins K (2013) Methodology for fitting and updating predictive accident models with trend. Accident Analysis and Prevention 56: 82-94, https://doi.org/10. 1016/j.aap.2013.03.009.

Cox DR (1983) Some remarks on overdispersion. Biometrika 70(1): 269-274, http://dx.doi.org/10.2307/2335966.

Dean C and Lawless JF (1989) Tests for detecting overdispersion in Poisson regression models. Journal of the American Statistical Association 84(406): 467-472, https://doi.org/10.1080/01621459.1989. 10478792.

Delen D, Sharda R and Bessonov M (2006) Identifying significant predictors of injury severity in traffic accidents using a series of artificial neural networks. Accident Analysis and Prevention 38(3): 434-444, https://doi.org/10.1016/j.aap.2005.06.024.

de Oña J, Mujalli RO and Calvo FJ (2011) Analysis of traffic accident injury severity on Spanish rural highways using Bayesian networks. Accident Analysis and Prevention 43(1): 402-411, http://dx.doi.org/10. 1016/j.aap.2010.09.010.

de Oña J, López G, Mujalli R and Calvo FJ (2013) Analysis of traffic accidents on rural highways using latent class clustering and Bayesian networks. Accident Analysis and Prevention 51: 1-10, http://dx.doi. org/10.1016/j.aap.2012.10.016.

Deublein M, Schubert M, Adey BT, Köhler J and Faber MH (2013) Prediction of road accidents: a Bayesian hierarchical approach. Accident Analysis and Prevention 51: 274-291, https://doi.org/10. 1016/j.aap.2012.11.019.

Deublein M, Schubert M and Adey BT (2014) Entwicklung, Anwendung und Evaluation einer Methode zur Prädiktion von Unfallrisiken auf Nationalstrassen (PURNA). Bundesamt für Strassen Astra, Ittigen, Switzerland (in German).

Deublein M, Schubert M, Adey BT and García de Soto B (2015) A Bayesian network model to predict accidents on Swiss highways. Infrastructure Asset Management 2(4): 145-158, https://doi.org/10. 1680/jinam.15.00008.

DFT (Department for Transport) (2011) Strategic Framework for Road Safety. DFT, London, UK. See https://assets.publishing.service.gov. uk/government/uploads/system/uploads/attachment_data/file/8146/ strategicframework.pdf (accessed 24/04/2017).

El-Basyouny K and Sayed T (2011) A full Bayes multivariate intervention model with random parameters among matched pairs for before-after safety evaluation. Accident Analysis and Prevention 43(1): 87-94, https://doi.org/10.1016/j.aap.2010.07.015.

ETSC (European Transport Safety Council) (2015a) Ranking EU Progress on Road Safety: 9th Road Safety Performance Index Report. ETSC, Brussels, Belgium. See http://etsc.eu/wp-content/uploads/ETSC-9thPIN-Report_Final.pdf (accessed 28/05/2016).

ETSC (2015b) UN to Back 2020 Goal to Halve Road Deaths and Injuries Worldwide. ETSC, Brussels, Belgium. See http://etsc.eu/un-to-back2020-goal-to-halve-road-deaths-and-injuries-worldwide/ (accessed on 31/07/2016)

García de Soto B (2014) A Methodology to Make Accurate Preliminary Estimates of Construction Material Quantities for Construction 
Infrastructure Asset Management

Volume 5 Issue 4
Predicting road traffic accidents using

artificial neural network models

García de Soto, Bumbacher, Deublein and Adey
Projects. PhD thesis, Swiss Federal Institute of Technology in Zurich (ETH Zürich), Zurich, Switzerland.

García de Soto B, Adey BT and Fernando D (2014) A process for the development and evaluation of preliminary construction material quantity estimation models using backward elimination-regression and neural networks. Journal of Cost Analysis and Parametrics 7: 1-39, http://dx.doi.org/10.1080/1941658X.2014.984880.

Gelman A, Carlin JB, Stern HS and and Rubin DB (2004) Bayesian Data Analysis. Chapman and Hall/CRC, Boca Raton, FL, USA.

GschloessI S and Czado C (2008) Modelling count data with overdispersion and spatial effects. Statistical Papers 49(3): 531-552, https://doi.org/10.1007/s00362-006-0031-6.

Hauer E (2001) Overdispersion in modelling accidents on road sections and in empirical Bayes estimation. Accident Analysis and Prevention 33(6): 799-808, https://doi.org/10.1016/S0001-4575(00)00094-4.

Hegazy T and Ayed A (1998) Neural network model for parametric cost estimation of highway projects. Journal of Construction Engineering and Management 124(3): 210-218, https://doi.org/10.1061/(ASCE) 0733-9364(1998)124:3(210).

Hegazy T, Fazio P and Moselhi O (1994) Developing practical neural network applications using back-propagation. Computer-Aided Civil and Infrastructure Engineering 9(2): 145-159, https://doi.org/10.1111/ j.1467-8667.1994.tb00369.x.

Hill T, Marquez L, O'Connor M and Remus W (1994) Artificial neural network models for forecasting and decision making. International Journal of Forecasting 10(1): 5-15, https://doi.org/10.1016/0169-2070 (94)90045-0.

Jadaan KS, Al-Fayyad M and Gammoh HF (2014) Prediction of road traffic accidents in Jordan using artificial neural network (ANN). Journal of Traffic and Logistics Engineering 2(2): 92-94, http://dx.doi.org/10. 12720/jtle.2.2.92-94

Jain AK, Mao J and Mohiuddin KM (1996) Artificial neural networks: a tutorial. Computer 29(3): 31-44, http://doi.ieeecomputersociety.org/10. $1109 / 2.485891$

Karlaftis M and Vlahogianni E (2011) Statistical methods versus neural networks in transportation research: differences, similarities and some insights. Transportation Research Part C: Emerging Technologies 19(3): 387-399, https://doi.org/10.1016/j.trc.2010.10.004.

Karlis D and Meligkotsidou L (2005) Multivariate Poisson regression with covariance structure. Statistics and Computing 15(4): 255-265, https:// doi.org/10.1007/s11222-005-4069-4.

Kim GH, An SH and Kang KI (2004) Comparison of construction cost estimating models based on regression analysis, neural networks, and case-based reasoning. Building and Environment 39(10): 1235-1242, https://doi.org/10.1016/j.buildenv.2004.02.013.

Kirby HR, Watson SM and Dougherty MS (1997) Should we use neural networks or statistical models for short-term motorway traffic forecasting? International Journal of Forecasting 13(1): 43-50, https://doi.org/10.1016/S0169-2070(96)00699-1.

Li H (1995) Neural networks for construction cost estimation. Building Research and Information 23(5): 279-284, https://doi.org/10.1080/ 09613219508727476.

Lord D and Mannering F (2010) The statistical analysis of crash-frequency data: a review and assessment of methodological alternatives. Transportation Research Part A: Policy and Practice 44(5): 291-305, https://doi.org/10.1016/j.tra.2010.02.001.

Mckim R (1993) Neural network applications to cost engineering. Cost Engineering, AACE 35(7): 31-35.

Miaou SP and Lord D (2003) Modeling traffic crash-flow relationships for intersections: dispersion parameter, functional form, and Bayes against empirical Bayes methods. Transportation Research Record: Journal of the Transportation Research Board 1840(1): 31-40, https://doi.org/10. 3141/1840-04

Mitra S and Washington S (2007) On the nature of over-dispersion in motor vehicle crash prediction models. Accident Analysis and Prevention 39(3): 459-468, https://doi.org/10.1016/j.aap.2006.08.002.

Motulsky HJ and Christopoulos A (2003) Fitting Models to Biological Data Using Linear and Nonlinear Regression: a Practical Guide to Curve Fitting. GraphPad Software Inc, San Diego, CA, USA.

Ogwueleka FN, Misra S, Ogwueleka TC and Fernandez-Sanz L (2014) An artificial neural network model for road accident prediction: a case study of a developing country. Acta Polytechnica Hungarica 11(5): 177-197.

Olden JD and Jackson DA (2002) Illuminating the 'black box': understanding variable contributions in artificial neural networks. Ecological Modelling 154: 135-150, http://dx.doi.org/10.1016/j. ecolmodel.2004.03.013.

Principe JC, Euliano NR and Lefebvre WC (2000) Neural and Adaptive Systems: Fundamentals Through Simulations. Wiley, New York, NY, USA.

Sameen MI and Pradhan B (2017) Severity prediction of traffic accidents with recurrent neural networks. Applied Sciences 7(6): 476-492, http:// dx.doi.org/10.3390/app7060476.

Xie Y, Lord D and Zhang Y (2007) Predicting motor vehicle collisions using Bayesian neural network models: an empirical analysis. Accident Analysis and Prevention 39(5): 922-933, https://doi.org/10.1016/j.aap. 2006.12.014.

Yeh IC (1998) Quantity estimating of building with logarithm-neuron networks. Journal of Construction Engineering and Management 124(5): 374-380, https://doi.org/10.1061/(ASCE)0733-9364(1998) 124:5(374).

Zhang G, Patuwo BE and Hu MY (1998) Forecasting with artificial neural networks: the state of the art. International Journal of Forecasting 14(1): 35-62, https://doi.org/10.1016/S0169-2070(97)00044-7.

\section{How can you contribute?}

To discuss this paper, please submit up to 500 words to the editor at journals@ice.org.uk. Your contribution will be forwarded to the author(s) for a reply and, if considered appropriate by the editorial board, it will be published as a discussion in a future issue of the journal. 